\title{
Photocatalytic Degradation of Methylene Blue Using Silver Nanoparticles Synthesized from Gymnema Sylvestre and Antimicrobial Assay
}

\author{
Murali Santhosh Kumar, Supraja N and David E*
}

Department of Biotechnology, India

\section{ISSN : 2688-836X}

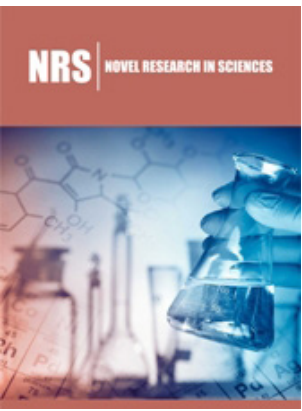

*Corresponding author: E David, Department of Biotechnology, India

Submission: 侮 July 22, 2019

Published: 㭗September 25, 2019

Volume 2 - Issue2

How to cite this article: Murali Santhosh Kumar, Supraja N, David E. Photocatalytic Degradation of Methylene Blue Using Silver Nanoparticles Synthesized from Gymnema Sylvestre and Antimicrobial Assay. Nov Res Sci.2(2). NRS.000532.2019. DOI: 10.31031/NRS.2019.2.000532

Copyright@ David E, This article is distributed under the terms of the Creative Commons Attribution 4.0 International License, which permits unrestricted use and redistribution provided that the original author and source are credited.

\begin{abstract}
The present study reports about the catalytic degradation of methylene blue (MB) in presence of Gymnema sylvestre mediated synthesized silver nanoparticles (AgNPs). AgNPs was produced from green synthesis method using aqueous extract. Formation of AgNPs from the synthesis was confirmed by using UV-visible spectroscopy with the appearance of surface plasmon (SPR) bands around $370 \mathrm{~nm}$. Functional groups were identified by using FT-IR, the particle size and zeta potential of AgNPs concluded by DLS, the agglomeration in microstructure of biosynthesized AgNPs was revealed by field scanning electron microscope (SEM). The synthesized of Ag NPs showed three diffraction peaks at $2 \theta$ values of $36.62^{\circ}$, $42.51^{\circ}$ and $65.84^{\circ}$ which corresponds to the (111), (200) and (220) planes of face-centered cubic. The AgNPs shown very good antimicrobial Assay towards microbes isolated from contaminated dye soil. The photocatalytic activity of the synthesized silver nanoparticles was examined by degradation of methylene blue under sunlight irradiation. Green synthesized silver nanoparticles were effectively degrading the dye nearly $95 \%$ at $7 \mathrm{~h}$ of exposure time.
\end{abstract}

Keywords: Gymnema sylvestre; AgNPs; Photocatalytic activity; Dye degradation; Antimicrobial assay

\section{Introduction}

Nanotechnology deals with the synthesis of nanoparticles with controlled size, shape, and dispersity of materials at the nanometer scale length and their potential use for human well-being. Nanometer sized materials have a high surface area; and a high fraction of surface atoms have been studied because of their exclusive properties such as optic, electronic, and catalytic Among all nanoparticles noble metal nanoparticles have enormous applications in diverse areas such as bioimaging, sensor, diagnosis, and novel therapeutic in biomedical field. Metallic silver and silver nanoparticles were recently applied as antimicrobial agents in various products such as cosmetics, animal feed coating of catheters, wound dressing and water purification with a minimal risk of toxicity in humans. Nowadays the biological systems were eagerly used for nanoscale material synthesis and assembly is an alternative method of physical and chemical process. Green approach of nanoparticles synthesis by biological entities has been gaining great advantages which are environmental benign, less toxic, and time consuming and, it is a single step process. Currently, plant and plant derived materials are used for nanoparticles synthesis which is more compatible than the microbe-mediated nanoparticles synthesis process because they eliminate the culture maintenance and are easy to handle. Nanoparticles synthesis by medicinal plants shows more benefit; they may enhance the antibacterial activity of silver nanoparticles, because the medicinally valuable active biomolecule present in the plants may bind on the surface of the nanoparticles and reduce the silver ions to silver nanoparticles Prasad \&Elumalai [1]. Nanoparticles can be organized into four types namely carbon based, metal based, dendrimers and composites nanoparticles are also referred to as organic nanoparticles. Spherical and ellipsoidal carbon nanoparticles are called as fullerenes, while cylindrical ones are called nanotubes. Quantum dots, nanogold, Nano silver and metal oxides (titanium oxide) are examples of metal-based nanoparticles. Dendrimers are Nano sized polymers which can be synthesized to perform specific chemical functions. Composites are c nanoparticles with other nanoparticles or with larger sized and heavier compounds. Silver nanoparticles are most commonly used in water filters and biosensors Patil et al. [2]; Kumar et al. [3]. 
Gymnema sylvestre is a perennial woody vine that grows in tropical areas of India, Africa, and Australia and has been used for medicinal purposes in Ayurvedic medicine. Common names include gymnema, Australian cowplant, and Periploca of the woods, and the Hindi term gurmar which means "sugar destroyer". The leaves and extracts contain Gymnema acids, the major bioactive constituents that interact with taste receptors on the tongue to temporarily suppress the taste of sweetness Supraja et al. [4]. Dyes are defined as colored, ionizing and aromatic organic compounds which show affinity towards the substrate to which it is being applied and they are extensively utilized in the textile industry Nagati et al. [5]; Bonnia et al. [6]. These non-biodegradable substances must be removed from the environment and pose to be a dire environmental crisis. Many methods are regularly used for reducing dyes like activated carbon sorption, flocculation, electro coagulation, UV-light degradation and redox treatments Vanaja et al. [7]. However, due to the ineptitude of these methods in one way or the other, the present situation necessitates bet and improved removal methods. Lately, studies have found that Ag nano particles are good, highly efficient and stable catalysts under ambient temperature with visible light illumination for degrading organic compounds and dyes Hence, the purpose of the present study was to assess the degrading property of the synthesized silver nanoparticles from Gymnema sylvestre leaves extract towards methylene blue and Antimicrobial Assay.

\section{Materials and Methods}

\section{Collection of leaves and preparation of Ag NPs}

Leaves of Gymnema sylvestre were collected from the Acharya N G Ranga Agricultural University, Tirupati, Andhra Pradesh, India. $10 \mathrm{~g}$ wet weight of fresh leaves was cut into fine pieces and washed with distilled water and boiled with $100 \mathrm{~mL}$ of double distilled water for $10 \mathrm{~min}$ at $60{ }^{\circ} \mathrm{C}$. Boiled mixture was filtered through Whatman No. 1 filter paper and collects the supernatant of leaf extract to that $90 \mathrm{ml}$ of silver nitrate solution is added left for overnight when there is a color changes from color less to brown indicate the formation of AgNPs and remaining leaf solution stored at $4{ }^{\circ} \mathrm{C}$ for further studies.

\section{Collection of dye sample}

The Dye sample were collected from Tirupur industry from Tirupur District in Tamilnadu, India. These dye samples were sended to Biotechnology department, Thiruvalluvar University, Vellore and the microbes were collected from dye soil samples through serial dilution and pure microbes were isolated by using streaking method in nutrient agar medium for bacteria and potato dextrose agar for fungi.

\section{Characterization of silver nanoparticles}

The UV-Vis spectrum of this solution was recorded in spectra 50 ANALYTIKJENA Spectrophotometer, which was operated in the wavelength range of $400-800 \mathrm{~nm}$. The FT-IR spectrum was taken in the mid IR region of $400-4000 \mathrm{~cm}^{-1}$. The spectrum was recorded using attenuated total reflectance technique. The crystalline structure of the nanoparticles was determined using the XRD technique. The XRD pattern was recorded using computer controlled XRD-system, JEOL, and Model: JPX-8030 with $\mathrm{Cu} \mathrm{K} \alpha$ radiation (Ni filtered $=13,418 \mathrm{~A}^{\circ}$ ) at the range of $40 \mathrm{kV}, 20 \mathrm{~A}$. The aqueous suspension of the synthesized nanoparticles was filtered through a $0.22 \mathrm{~lm}$ syringe driven filter unit, and the hydro dynamic diameter (HDD) of the distributed nanoparticles was measured by the principle of dynamic light scattering (DLS) using Nanopartica (HORIBA, SZ-100) compact scattering spectrometer, the surface morphological studies of the AgNPs samples were carried out with scanning electron microscope (SEM) (Hitachi's SU6600) at magnification ranging from 10 to 600,0000 operated at an accelerating voltage of $30 \mathrm{kV}$.

\section{Photocatalytic degradation of dye}

Typically, $10 \mathrm{mg}$ of methylene blue dye was added to $1000 \mathrm{~mL}$ of double distilled water used as stock solution. About $10 \mathrm{mg}$ of biosynthesized silver nanoparticles was added to $100 \mathrm{~mL}$ of methylene blue dye solution. A control was also maintained without addition of silver nanoparticles. Before exposing to irradiation, the reaction suspension was well mixed by being magnetically stirred for $30 \mathrm{~min}$ to clearly make the equilibrium of the working solution. Afterwards, the dispersion was put under the sunlight and monitored from morning to evening sunset. At specific time intervals, aliquots of $2-3 \mathrm{~mL}$ suspension were filtered and used to evaluate the photocatalytic degradation of dye. The absorbance spectrum of the supernatant was subsequently measured using UVVis spectrophotometer at the different wavelength. Concentration of dye during degradation was calculated by the absorbance value at $650 \mathrm{~nm}$ Supraja et al. [8].

\section{Antimicrobial activity of plant biosynthesized silver nanoparticles}

The antimicrobial activity of Gymnema sylvestre leaf extracts silver nanoparticles was examined based on colony formation by in-vitro Petri dish assays (disc diffusion). Each fungal and bacterial isolate was cultured on growth media that induced prolific conidia and bacterial production. The fungus isolates were grown on potato dextrose agar medium, and bacterial isolates were grown on nutrient agar medium. Conidia were collected from cultures that were incubated at $37{ }^{\circ} \mathrm{C}$ for 10 days (fungi), and bacterial cultures were collected from cultures that were incubated at $37^{\circ} \mathrm{C}$ for 2 days for (bacteria) and diluted with sterile, deionized water to a concentration of 106 spore'sml $^{-1}$. Aliquots of the conidial suspension and bacterial suspension were mixed with serial concentrations of silver preparations to a final volume of $1 \mathrm{ml}$ and were also mixed with sterile, deionized water as control. A $10 \mu \mathrm{l}$ subsample of the conidia and Gymnema sylvestre silver mixture stock was taken at $30 \pm 0.8,100 \pm 1.1$ and $170 \pm 1.4 \mathrm{ppm}$ after silver treatments and diluted 100-foldwith the deionized water. A $10 \mu$ l aliquot of the diluted spore suspension was spread on PDA (Becton, Dickson and Company, Sparks, MD) medium. Three PDA plates for fungi and three NAplates for bacteria per each combination of exposure silver concentration were tested. The filter paper disc dipped in different ppm and inserted on mediums (PDA), and then, the plates were incubated at $37^{\circ} \mathrm{C}$ for 2-4 days for fungi and bacteria, respectively. The average number of colonies from silver-treated 
spore suspensions (fungi) and (bacteria) was compared with the number on the water control (percent colony formation). The zone size was determined by measuring the diameter of the zone in $\mathrm{mm}$ Aneja 2003; Supraja et al. [9].

\section{Statistical analysis}

All the data from three independent replicate trials were subjected to analysis using Statistical package for the Social Sciences (SPSS) version 16.0. The data are reported as the mean \pm SD.

\section{Result and Discussion}

UV-visible spectroscopy characterization method was then used to monitor formation of AgNPs synthesized from Gymnema sylvestre (Figure 1) and its properties. The absorption of light by the nanoparticles at different wavelengths provides an indication of particle size, while the breadth of the peaks signifies the particle size distribution. The characteristic SPR band of AgNPs is in the range of $370 \mathrm{~nm}$ Edeoga et al. [10]. The overall observations suggest that the bio-reduction of (silver ions) $\mathrm{Ag}(+)$ to $\mathrm{Ag}(0)$ was confirmed by UV-Vis spectroscopy (Figure 2).

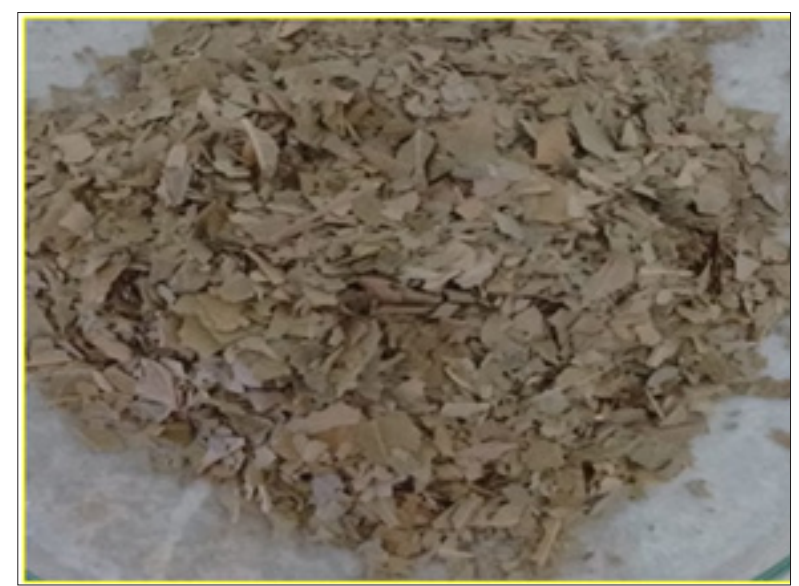

Figure 1: Gymnema sylvestre leaves.

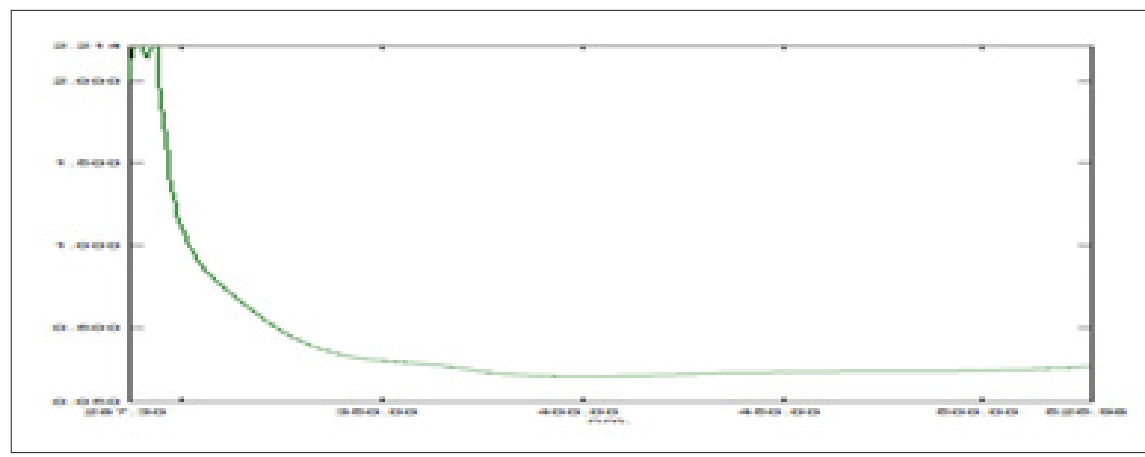

Figure 2: UV-Visible spectrum of Gymnema sylvestre leaf extract mediated synthesized silver nanoparticles.

FT-IR spectrum of the biosynthesized silver nanoparticles using Gymnema sylvestre leaf extract (Figure 3) The peak present at $1982 \mathrm{~cm}^{-1}$ reveals the presence of $\mathrm{C}-\mathrm{H}$ stretching vibration of alkynes, $1796 \mathrm{~cm}^{-1}$ reveals the presence of $\mathrm{C}-\mathrm{H}$ stretching vibration of alkanes, $1388 \mathrm{~cm}^{-1}$ reveals the presence of $\mathrm{C}-\mathrm{H}$ stretching vibration of aldehydes, 872 and $713 \mathrm{~cm}^{-1}$ reveals the presence of
C-Br stretching vibration of alkyl halides. The nanoparticles are bound to the functional organic groups (carboxyl and amine) from the Gymnema sylvestre leaf extract, and these functional groups may act as template, reducing and capping agents of silver nanoparticles Arun et al. [11].

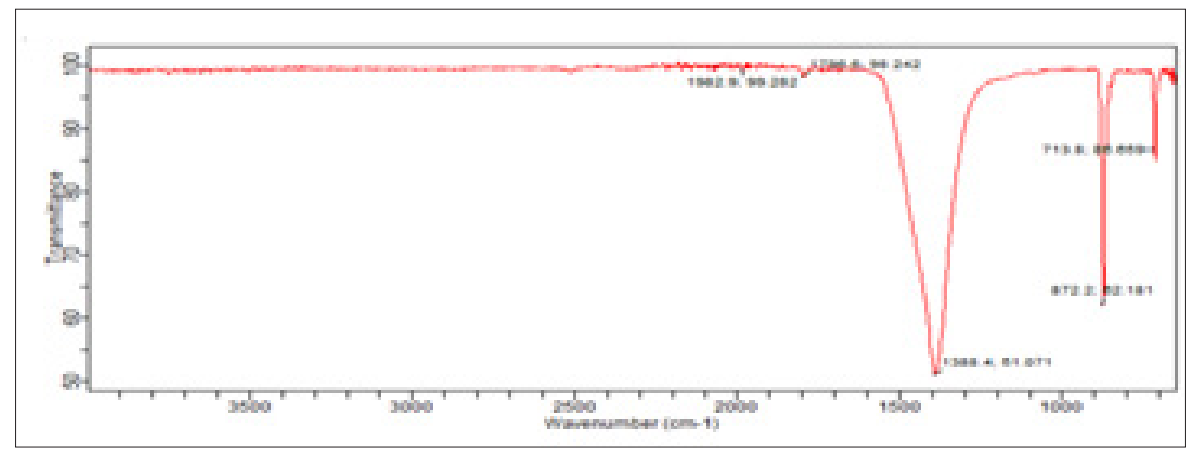

Figure 3: FT-IR spectrum of Gymnema sylvestre leaf extract mediated synthesized silver nanoparticles.

The powder XRD pattern was recorded for the identification of phases exhibited by the biosynthesized AgNPs. The synthesized of AgNPs showed five diffraction peaks (Figure 4) at $2 \theta$ values of $36.62^{\circ}, 42.51^{\circ}$ and $65.84^{\circ}$ which corresponds to the (111), (200) and (220) planes of face-centered cubic the peak corresponding to the (111) plane is more intense than that of other planes suggesting the predominant growth of AgNPs along (111) direction Supraja et al. [8]. 


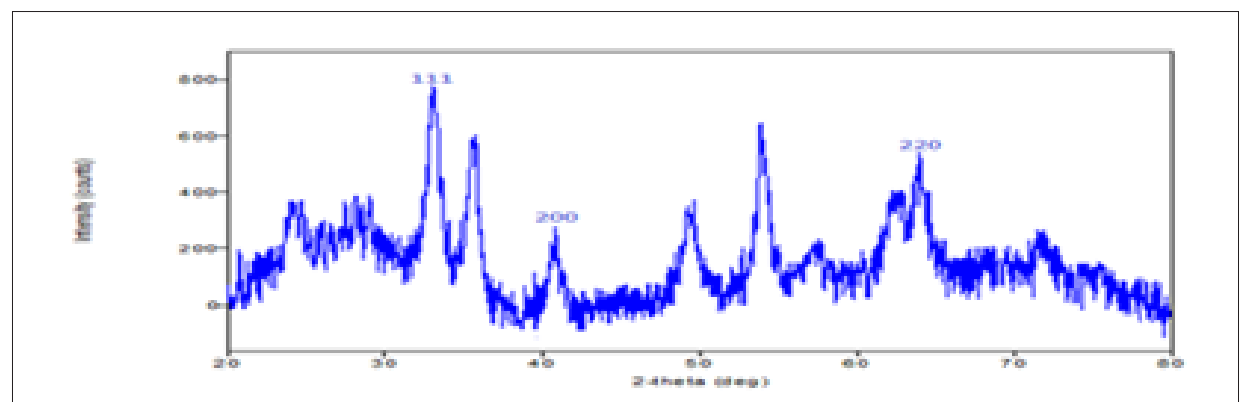

Figure 4: XRD spectrum of Gymnema sylvestreleaf extract mediated synthesized silver nanoparticles.

Particle size and zeta potential values were measured using Nanopartica SZ-100 (HORIBA). The zeta potential spectra for the Gymnema sylvestre leaf extract nanoparticles were recorded zeta potential verses intensity spectra with zeta potential $(\mathrm{mV})$ on $\mathrm{x}$-axis and intensity (a.u) on y-axis particle size of $95.2 \mathrm{~nm}$ and zeta potential of $100.2 \mathrm{mV}$ were recorded (Figure $5 \mathrm{a} \& 5 \mathrm{~b}$ ), Here the zeta potential value of the silver nanoparticles indicated good stability with high potential Sri Sindhura et al. [12].

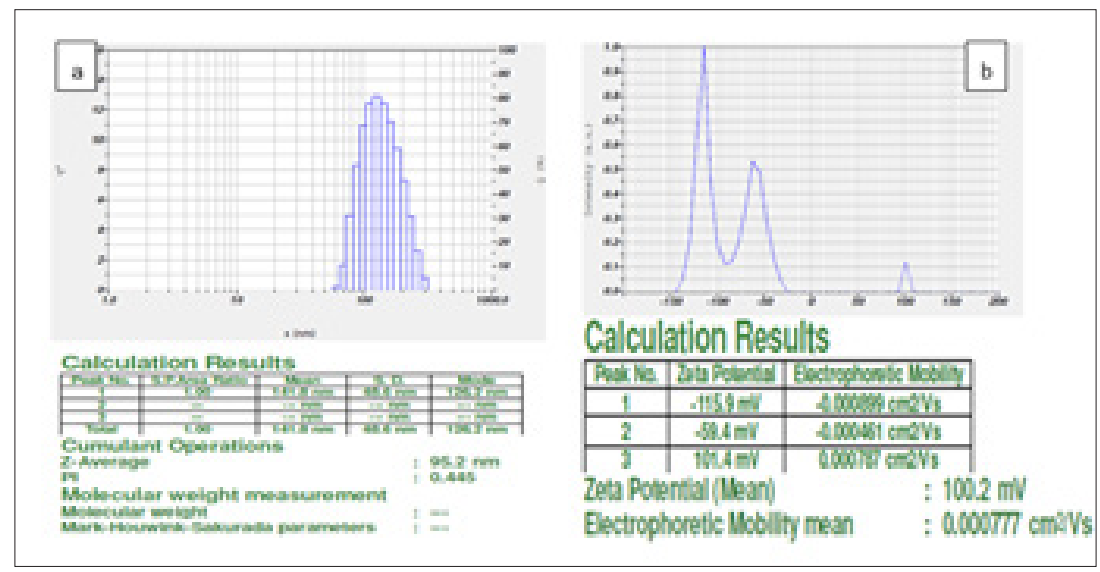

Figure 5: DLS spectrum

(a) Particle size (b) Zeta potential of Gymnema sylvestre leaf extract mediated synthesized silver nanoparticles.

SEM image shows the size and shape of the biosynthesized silver nanoparticles using Gymnema sylvestre leaf extract. Size of the nanoparticles was observed at different magnifications. Spherical and rhombohedral shape of nanoparticles with high agglomeration was noted with the size range from $1 \mu \mathrm{m}$ to $200-400 \mathrm{~nm}$ (Figure
6). Polydispersed nanoparticles were observed in SEM image and revealed the result of UV-Vis spectrophotometer. The surfaces of aggregated nanoparticles were shown to be rough. Aggregation of nanoparticles took place due to the insufficiency of capping agent in the leaf extract to synthesis of nanoparticles Prabha et al. [13].
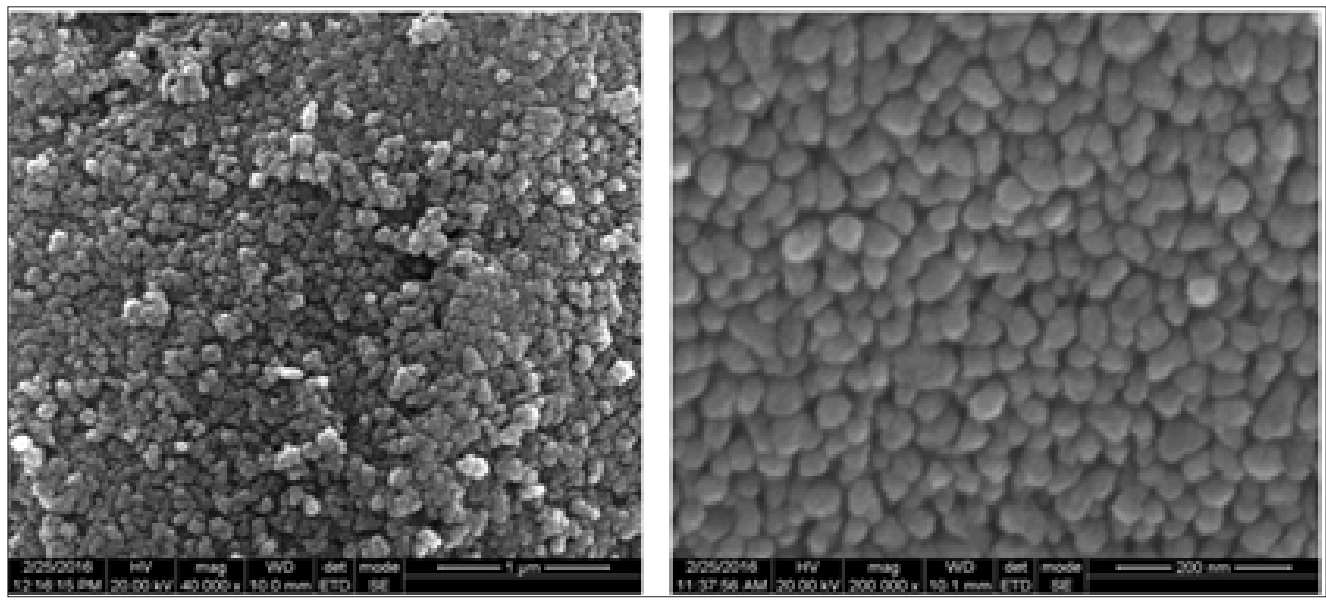

Figure 6: SEM analysis of Gymnema sylvestre leaf extract mediated synthesized silver nanoparticles 


\section{Photocatalytic degradation of dye}

Photocatalytic degradation of methylene blue was carried out by using green synthesized silver nanoparticles under solar light. Dye degradation was initially identified by color change. Initially, the color of dye shows deep blue color changed into light blue after the $1 \mathrm{~h}$ of incubation with silver nanoparticles while exposed to solar light (Figure $7 \& 8$ ). Thereafter light blue was changed into light green. Finally, the degradation process was completed at 7 hand was identified by the change of reaction mixture color to colorless.
The adsorption of Ag nanoparticles on the methylene blue solution was initially low and further increased with constant increase in time and the percentage of dye degradation. Altogether, the photocatalytic properties of Ag nanoparticles in visible light may be well due to excitation of Surface Plasmon Resonance, However, at the same time more photo-catalyst would also have induced greater aggregation, which resulted in the decrease in the surface area of the photo-catalyst, making a significant fraction of the catalyst to be inaccessible to adsorbing the dye Vanaja et al. [7].

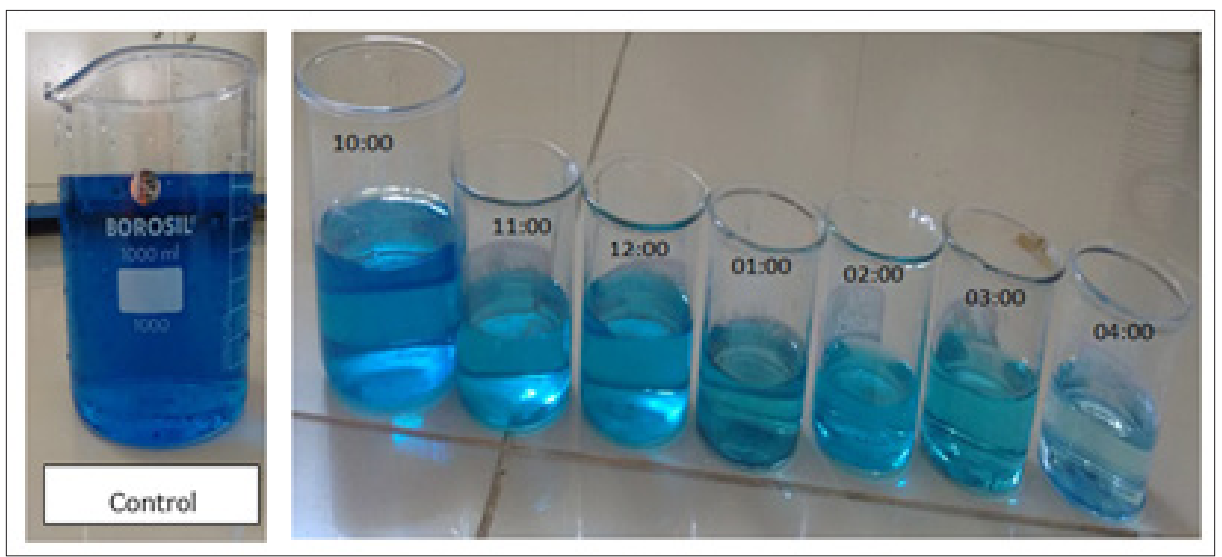

Figure 7: Photo-catalytic degradation of methylene blue using Gymnema sylvestre leaf extract mediated synthesized silver nanoparticles.

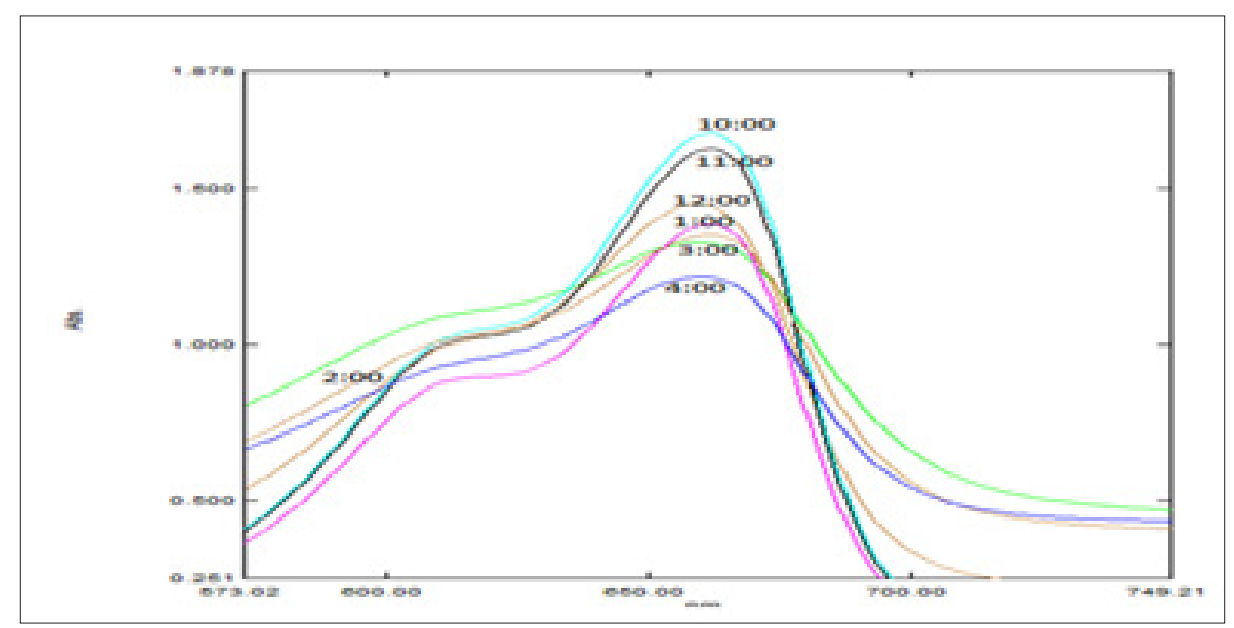

Figure 8: Graph showing UV-visible spectrum of different time intervals of Photo-catalytic degradation of methylene blue using Gymnema sylvestre leaf extract mediated synthesized silver nanoparticles.

\section{Antimicrobial assay}

Silver nanoparticles obtained from Gymnema sylvestre leaf extract have shown very strong inhibitory action against fungal $s p$, Gram-positive and Gram-negative bacteria (Figure 9\&10). Three concentrations of AgNPs (170,100,50ppm) were prepared and were applied against an array of fungal species viz, Aspergillus niger, Rhizopus stolonifer, Aspergillus flavus, Fusarium oxysporium and bacterial species viz, E. Coli, Bacillus pantothenticus, Pseudomonas fluorescence and Salmonella typhimurium. The higher concentration (170ppm) of leaf mediated synthesized AgNPs showed significant antimicrobial effect (Table 1\&2) compared with other concentrations $(100,50 \mathrm{ppm})$ seen in leaf, but when compared to bacterial concentrations fungi shown very good antimicrobial activity towards all concentrations. The inhibitory action of the microbes may be attributed to the loss of replication ability of DNA upon treatment with the silver ion, besides the fact that expression of ribosomal sub-unit proteins as well as some other cellular proteins and enzymes essential to ATP production becomes inactivated Kouvaris et al. [14]. But to understand the mechanisms of action of these agents, more detailed chemical structure elucidation of the bioactive components followed by therapeutic investigations and toxicological assessment are required. 


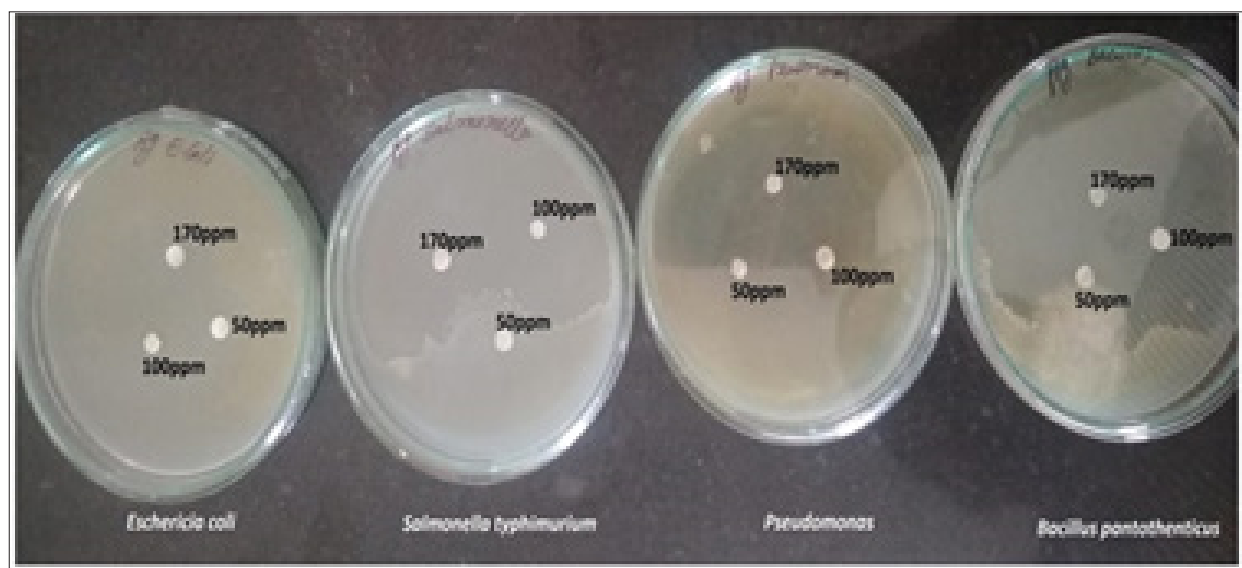

Figure 9: Antibacterial activity of different concentrations (50,100,170ppm) of Gymnema sylvestre leaf extract mediated synthesized silver nanoparticles.

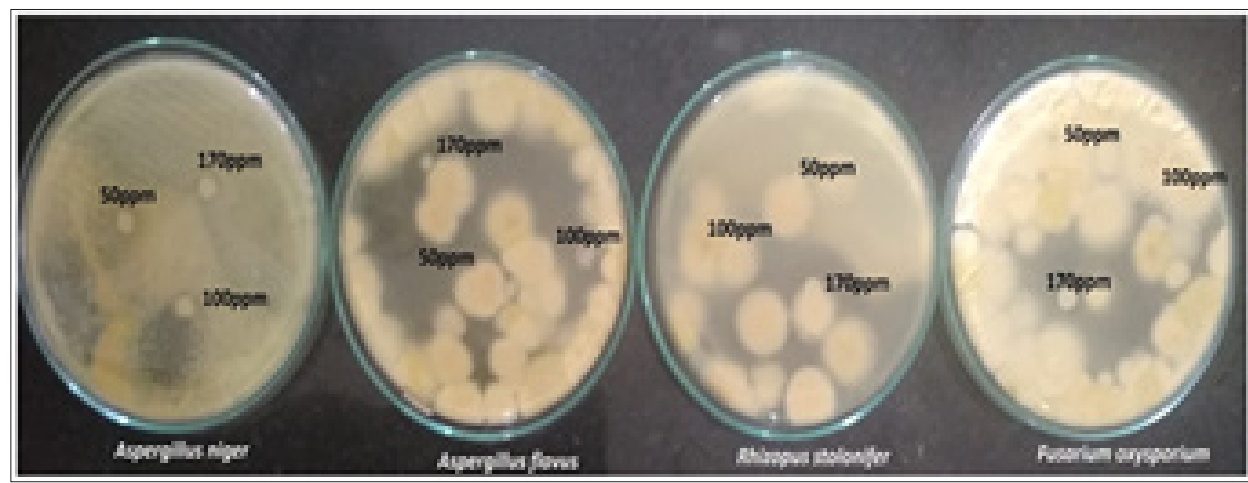

Figure 10: Antifungal activity of different concentrations (50,100,170ppm) of Gymnema sylvestre leaf extract mediated synthesized silver nanoparticles.

Table 1: In-vitro antibacterial studies against bacterial pathogens using Gymnema sylvestre leaf extract mediated synthesized silver nanoparticles.

\begin{tabular}{|c|c|c|c|c|}
\hline S. No & Bacteria Names & $\mathbf{1 7 0} \mathbf{p p m} \pm \mathbf{1 . 4} \mathbf{p p m}$ & $\mathbf{1 0 0 p p m} \pm \mathbf{1 . 1} \mathbf{p p m}$ & $\mathbf{5 0 p p m} \pm \mathbf{0 . 8} \mathbf{p p m}$ \\
\hline 1 & Escherichia coli & $1.3 \pm 0.07$ & $0.9 \pm 0.05$ & $0.5 \pm 0.03$ \\
\hline 2 & Salmonella typhimurium & $1.8 \pm 0.08$ & $1.3 \pm 0.06$ & $1.0 \pm 0.02$ \\
\hline 3 & Pseudomonas & $1.2 \pm 0.1$ & $0.8 \pm 0.09$ & $0.5 \pm 0.07$ \\
\hline 4 & Bacillus pantothenticus & $2.6 \pm 0.4$ & $1.8 \pm 0.2$ & $1.2 \pm 0.09$ \\
\hline
\end{tabular}

Table 2: In-vitro antifungal studies against fungal pathogens using Gymnema sylvestre leaf extract mediated synthesized silver nanoparticles..

\begin{tabular}{|c|c|c|c|c|}
\hline S. No & Fungi names & $\mathbf{1 7 0} \mathbf{p p m} \pm \mathbf{1 . 4} \mathbf{p p m}$ & $\mathbf{1 0 0 p p m} \pm \mathbf{1 . 1} \mathbf{p p m}$ & $\mathbf{5 0 p p m} \pm \mathbf{0 . 8 p p m}$ \\
\hline 1 & Aspergillus Niger & $2.5 \pm 0.6$ & $2.1 \pm 0.4$ & $1.4 \pm 0.08$ \\
\hline 2 & Aspergillus flavus & $4.2 \pm 0.9$ & $3.5 \pm 0.7$ & $1.6 \pm 0.5$ \\
\hline 3 & Rhizopus stolonifer & $3.1 \pm 0.9$ & $2.4 \pm 0.6$ & $2.2 \pm 0.3$ \\
\hline 4 & Fusarium oxysporium & $4.0 \pm 0.9$ & $3.7 \pm 0.8$ & $3.0 \pm 0.5$ \\
\hline
\end{tabular}

\section{Conclusion}

Green nanotechnology is gaining importance due to the elimination of harmful reagents and provides effective synthesis of expected products in an economical manner. Green synthesis of silver nanoparticles shows more compatible, ecofriendly, low cost, and less time-consuming process. Herein, the silver nanoparticles were synthesized by using plant leaf extract of Gymnema sylvestre leaf extract, the photocatalytic activity of green synthesized silver nanoparticles was evaluated by choosing methylene blue dye. The main absorption peak at $650 \mathrm{~nm}$ decreased gradually with the extension of the exposure time indicating the photocatalytic degradation of methylene blue dye. The present study, it is found that the use of natural renewable and eco-friendly reducing agent used for synthesis of silver nanoparticles exhibits excellent 
photocatalytic activity against dye molecules and can be used in water purification systems and dye effluent treatment

\section{Acknowledgment}

Authors are thankful to Acharya N.G. Ranga Agricultural University for providing research facility at institute of Frontier Technology, Regional Agricultural Research Station, Tirupati to carry out this part of the research work.

\section{References}

1. Prasad TNVKV, Elumalai EK (2011) Bio fabrication of Ag nanoparticles using Moringa oleifera leaf extract and their antimicrobial activity. Asian Pacific Journal of Tropical Biomedicine 1(6): 439-442.

2. Patil RS, Kokate MR, Kolekar SS (2012) Bioinspired synthesis of highly stabilized silver nanoparticles using Ocimum tenuiflorum leaf extract and their antibacterial activity. Spectrochimica Acta A 91: 234-238.

3. Kumar R, Roopan SM, Prabhakarn A, Khanna VG, Chakroborty S (2012) Agricultural waste Annona squamosa peel extract: Biosynthesis of silver nanoparticles. Spectrochimica Acta A 90: 173-176.

4. Supraja N, Avinash B, Prasad TNVKV (2017) Green synthesis and characterization of silver nanoparticles from Gymnema sylvestre leaf extract: Study of antimicrobial activities. Int J Curr Microbiol App Sci 6(3): 530-540.

5. Nagati V, Koyyati R, Donda MR, Alwala J, Kundle KR, et al. (2012) Green synthesis and characterization of silver nanoparticles from Cajanuscajan leaf extract and its antibacterial activity. International Journal of Nanomaterials and Biostructures 2(3): 39-43.

6. Bonnia NN, Kamaruddin MS, Nawawi MH, Ratim S, Azlina HN, et al. (2016) Green biosynthesis of silver nanoparticles using Polygonum hydropiper and study its catalytic degradation of methylene blue. Procedia Chemistry 19: 594-602.

7. Vanaja M, Paulkumar K, Baburaja M, Rajeshkumar S, Gnanajobitha G, et al. (2014) Degradation of methylene blue using biologically synthesized silver nanoparticles. Bioinorg Chem Appl p. 8.

8. Supraja N, Prasad TNVKV, Giridhara Krishna T, David E (2016) Synthesis, characterization, and evaluation of the antimicrobial efficacy of Boswellia ovalifoliolata stem bark-extract-mediated zinc oxide nanoparticles. Appl Nanosci 6(4): 581-590.

9. Supraja N, Prasad TNVKV, Giridhara Krishna T, David E (2015) Synthesis, characterization and evaluation of the antimicrobial efficacy of Boswellia ovalifoliolata stem bark extract mediated zinc oxide nanoparticles. Appl Nanosci, Switzerland.

10. Edeoga HO, Okwu DE, Mbaebie BO (2005) Phytochemical constituents of some Nigerian medicinal plants. African J Biotechnol 4(7): 685-688.

11. Arun LB, Arunachalam AM, Arunachalam KD, Annamalai SK, Amit Kumar $\mathrm{K}$ (2014) In vivo antiulcer, anti-stress, anti-allergic, and functional properties of gymnemic acid isolated from Gymnema sylvestre R Br. BMC Complement Altern Med 14: 70.

12. Sindhura S, Prasad TNVKV, Panner SP, Hussain OM (2013) Synthesis and characterization of phytogenic zinc nanoparticles and their antimicrobial activity. Appl Nanosci 4(7): 819-827.

13. Prabha S, Supraja N, Garud M, Prasad TNVKV (2014) Synthesis, characterization and antimicrobial activity of Alstonia scholaris barkextract-mediated silver nanoparticles. J Nanostruct Chem 4(4): 161-170.

14. Kouvaris P, Delimitis A, Zaspalis V, Papadopoulos D, Tsipas SA, et al. (2012) Green synthesis and characterization of silver nanoparticles produced using Arbutus Unedo leaf extract. Mater Lett 76: 18-20. 